\title{
Infarto agudo de miocardio con elevación del segmento ST en la Argentina. Datos del registro continuo ARGEN-IAM-ST
}

\author{
Acute ST-segment Elevation Myocardial Infarction in Argentina. Data from the continuous \\ ARGEN-IAM-ST registry
}

\author{
HERALDO D'IMPERIO ${ }^{\oplus}$, JUAN GAGLIARDI ${ }^{\oplus}$, ADRIÁN CHARASK ${ }^{\oplus}$, RODRIGO ZONI ${ }^{\oplus}$, WALTER QUIROGA ${ }^{\oplus}$, YANINA CASTILLO COSTA ${ }^{\oplus}$, \\ GUSTAVO CEREZO, CARLOS TAJER ${ }^{\oplus}$. POR LOS INVESTIGADORES DE ARGEN IAM-ST
}

\begin{abstract}
RESUMEN
Introducción: A través del Registro Nacional de Infarto Agudo de Miocardio (IAM) con Elevación del ST (ARGEN IAM-ST) realizado en 2015 se conocieron datos de la realidad del IAM en Argentina; en esta ocasión, se presenta un reporte actual. Material y métodos: Estudio prospectivo multicéntrico, con alcance nacional. Luego de la primera fase de la encuesta ARGENIAM-ST, se invitó a los centros a continuar con el registro de IAM. Se incluyeron pacientes con IAM con elevación del segmento ST en el electrocardiograma de hasta 36 horas de evolución.

Resultados: La población analizada abarcó 2464 pacientes asistidos en 78 centros. La media de edad fue $60 \pm 12$ años y el $80 \%$ fue de sexo masculino. Los factores de riesgo prevenibles se distribuyeron del siguiente modo: tabaquismo $45 \%$, hipertensión arterial $58 \%$, diabetes $24 \%$ y dislipidemia $41 \%$. El $11 \%$ tuvo antecedente de enfermedad coronaria. El $88 \%$ recibió reperfusión; el $21 \%$ de estos pacientes recibieron trombolíticos y al $89 \%$ se le realizó angioplastia. La demora desde el inicio de los síntomas hasta la admisión fue de 130 minutos (RIC 25-75: 60-305); los médicos reportaron demoras hasta el tratamiento en el $49 \%$ de los casos, con impacto en los tiempos totales de isquemia (TTI). La mortalidad intrahospitalaria fue del 8,7\%. En el análisis multivariado, ser tratado en un centro con hemodinamia no se asoció de forma independiente con la supervivencia. Conclusiones: Los datos actuales del registro continuo de IAM en la Argentina son similares a los que mostró la encuesta de 2015. Las demoras hasta el tratamiento son importantes, especialmente por el tiempo de demora en la consulta de los pacientes, lo que impacta en gran medida en los TTI.
\end{abstract}

Palabras claves: Infarto de miocardio - Infarto de miocardio con elevación del ST - Epidemiología - Angioplastia coronaria con balón - Reperfusión

\begin{abstract}
Background: The National ST-segment elevation Acute Myocardial Infarction (ARGEN-AMI-ST) registry carried out in 2015 provided data on the reality of AMI in Argentina.

Objective: The aim of this study was to present an updated report of the ARGEN-AMI-ST registry.

Methods: This was a national, prospective, multicenter study. After the first phase of the ARGEN-AMI-ST survey, centers were invited to continue with the AMI registry including patients with up to 36-hour electrocardiographic STEMI evolution Results: The analyzed population comprised 2,464 patients assisted in 78 centers. Mean age was $60 \pm 12$ years and $80 \%$ were men. Preventable risk factors were: $45 \%$ smoking, 58\% hypertension, $24 \%$ diabetes, $41 \%$ dyslipidemia and $11 \%$ history of coronary heart disease. Eighty-eight percent of patients underwent reperfusion, and among them, $21 \%$ received thrombolytics and $89 \%$ percutaneous coronary intervention. The delay from onset of symptoms to admission was 130 minutes (IQR 25-75: 60-305); physicians reported delays to treatment in $49 \%$ of cases, with an impact on total ischemic times (TIT). In-hospital mortality was $8.7 \%$. In the multivariate analysis, being treated in a center with hemodynamic availability was not independently associated with survival.

Conclusions: Current data from the continuous AMI registry in Argentina are similar to those shown in the 2015 survey. Delays to treatment are important, especially due to the delay in patient consultation, which greatly impacts on TIT.
\end{abstract}

Key words: Myocardial infarction - ST segment elevation myocardial infarction - Epidemiology - Coronary balloon angioplasty - Reperfusion

\section{INTRODUCCIÓN}

Las enfermedades cardiovasculares son la principal causa de morbimortalidad global y tienen especial impacto en los países en desarrollo; es por ello que se han diseñado múltiples estrategias y programas cuyos objetivos son la prevención y el tratamiento de estas patologías, con el fin de disminuir sus consecuencias. (1)

Las sociedades científicas del ámbito cardiovascular en la Argentina, esto es, la Sociedad Argentina de Cardiología (SAC) y la Federación Argentina de Cardiología (FAC), tienen una larga trayectoria de 
registros y programas educativos para mejorar la asistencia cardiovascular. Para conocer cuáles son las medidas necesarias y sus resultados en la práctica, se deben tener registros de prevalencia y/o incidencia de los eventos, a fin de administrar los recursos y permitir que estos se distribuyan de manera equitativa. (2)

El infarto agudo de miocardio (IAM) con elevación del ST (IAMCEST) es una de las patologías hacia las que se destinan estos recursos, ya que su atención y tratamiento oportuno reducen la mortalidad cardiovascular y disminuyen las complicaciones en el corto y largo plazo, con un beneficio claro en la población general.

Es por esto que en un trabajo de colaboración entre la SAC y la FAC, en el año 2015 se implementó la Encuesta Nacional de Infarto con Elevación del ST (ARGEN-IAM-ST), con el objetivo de relevar a nivel nacional la atención de IAMCEST en nuestro país; los resultados de ese análisis ya han sido publicados. (3)

Debido a la importancia de estos datos y la aceptación de los centros participantes a continuar con la colaboración, el proyecto avanzó con el registro continuo de IAMCEST, gracias al cual se cuenta con información actualizada e ininterrumpida acerca de esta patología en la Argentina.

\section{MATERIAL Y MÉTODOS}

Se trata de un estudio prospectivo multicéntrico, de alcance nacional. Finalizada la primera fase del Registro Nacional de Infarto Agudo de Miocardio con Elevación del ST (ARGENIAM-ST), en diciembre de 2015, se invitó a los centros participantes a continuar con el registro; con este objetivo se realizó una adecuación de la ficha de casos, en la que permanecieron los datos considerados de mayor relevancia epidemiológica y clínica.

Se mantuvieron los criterios de inclusión y exclusión que fueron publicados previamente. (3) Se recabaron datos de las características del paciente (edad, género, factores de riesgo, antecedentes, comorbilidades), del cuadro clínico (localización del infarto, Killip y Kimbal de ingreso, tiempo de evolución), del tratamiento empleado (antiagregantes, de reperfusión, tratamiento coadyuvante) y de la evolución clínica intrahospitalaria (insuficiencia cardíaca, angina posinfarto, shock, muerte). Se obtuvieron los datos relacionados con las demoras hasta lograr un tratamiento efectivo.

Se consideraron los siguientes tiempos y demoras:

1) Tiempo dolor-consulta: tiempo transcurrido entre el inicio de los síntomas sugestivos de isquemia coronaria y el primer contacto médico.

2) Tiempo a la reperfusión: tiempo transcurrido entre el arribo a un centro médico y el inicio de tratamiento de reperfusión:

a) En caso de fibrinolíticos:

- Tiempo ventana: intervalo de tiempo en minutos desde el inicio de los síntomas hasta el comienzo de la infusión.

- Tiempo puerta-aguja: intervalo de tiempo en minutos desde el arribo a la institución y el comienzo de la infusión.

b) En caso de angioplastia:

- Tiempo ventana: intervalo de tiempo en minutos desde el inicio de los síntomas hasta el insuflado del balón.
- Tiempo puerta-balón: intervalo de tiempo en minutos desde el arribo a la institución hasta el insuflado del balón.

La recolección de los datos se realizó vía web, en una ficha de formato electrónico especialmente diseñada por el Centro de Teleinformática Médica de la FAC (CETIFAC).

\section{Consideraciones éticas}

El protocolo del registro ARGEN IAM-ST fue aprobado por el comité de ética de la Sociedad Argentina de Cardiología y el de cada institución participante.

\section{Análisis estadístico}

Las variables cualitativas se presentan como frecuencias y porcentajes, con sus intervalos de confianza. Para la descripción de las variables cuantitativas, se utilizó la media \pm el desvío estándar (DE) o la mediana y el rango intercuartilo (RIC 25-75), según su distribución.

El análisis de las variables discretas se realizó a través de tablas de contingencia y el de las variables continuas, por el test de t o Kruskall Wallis para datos no apareados o mediante el análisis de la varianza (ANOVA), según correspondiera. Se realizó análisis de correlación para evaluar asociaciones. Se consideró significativo un valor de $\mathrm{p}<0,05$. El análisis se realizó con Stata/SE v13.0 ${ }^{\circledR}$.

El protocolo fue registrado en ClinicalTrials.gov con el número NCT2458885.

\section{RESULTADOS}

Desde el 1 de enero de 2016 hasta el 10 de septiembre de 2019 se incluyeron 2464 pacientes atendidos en 78 centros de 19 provincias del país y la Ciudad Autónoma de Buenos Aires (42\% entidades públicas y 58\% privadas). El $73 \%$ de los pacientes correspondieron a unidades coronarias exclusivas y el $65 \%$ a centros con capacidad de realizar angioplastia.

La media de edad de los pacientes incluidos fue de $60 \pm 12$ años y el $80 \%$ fue de sexo masculino. Del total de pacientes que ingresaron al registro, solo $28(1,1 \%)$ lo hicieron con bloqueo de rama izquierda.

La prevalencia de los factores de riesgo coronarios prevenibles fue la siguiente: tabaquismo activo $45 \%$, hipertensión arterial esencial 58\%, diabetes $24 \%$ y dislipidemia $41 \%$. El 13\% de los pacientes presentó antecedentes de enfermedad coronaria.

La localización del infarto fue anterior en el 37\% de los casos, inferior en el $38 \%$ y lateral en el $4,4 \%$. Al ingreso, 342 pacientes $(13,9 \%)$ presentaron signos de insuficiencia cardíaca, mientras que la presencia de shock cardiogénico al ingreso fue del 7,5\%. Las características de la población se resumen en la Tabla 1.

\section{Tratamiento de reperfusión}

La mitad de los casos ingresó a una institución donde fueron tratados, en primera instancia, para el IAM; la otra mitad arribó a un centro que transfirió al paciente a otro nivel de complejidad para su atención según la organización local.

El $88 \%$ del total de los pacientes $(\mathrm{n}=2178)$ recibieron reperfusión; 443 de esos pacientes $(21 \%)$ fueron reperfundidos con trombolíticos. Se realizó angioplastia 
Tabla 1. Características generales

\begin{tabular}{|c|c|c|c|}
\hline Variable & $\mathbf{n}$ & $\%$ & IC $95 \%$ \\
\hline Edad (media $\pm \mathrm{DE}^{*}$ ) & $60 \pm 12$ & & \\
\hline Género masculino & 1973 & 80 & $79-81$ \\
\hline \multicolumn{4}{|l|}{ Factores de riesgo coronario } \\
\hline Hipertensión arterial & 1426 & 58 & $56-60$ \\
\hline Desconoce & 120 & 5 & $4-6$ \\
\hline Diabetes & 589 & 24 & $22-26$ \\
\hline Desconoce & 102 & 4 & $4-5$ \\
\hline Dislipidemia & 1006 & 41 & $39-43$ \\
\hline Desconoce & 310 & 13 & $11-14$ \\
\hline Tabaquismo & 1110 & 45 & $43-47$ \\
\hline Extabaquismo & 592 & 24 & $22-26$ \\
\hline Antec. familiares & 444 & 18 & $16-20$ \\
\hline \multicolumn{4}{|l|}{ Antecedentes coronarios } \\
\hline Infarto & 269 & 11 & $10-12$ \\
\hline Antec. enfermedad coronaria & 317 & 13 & $11-14$ \\
\hline Angina crónica estable & 83 & 3,4 & $2,7-4$ \\
\hline Angioplastia & 241 & 10 & $9-11$ \\
\hline CRM & 39 & 1,7 & $1,1-2,1$ \\
\hline Historia de insuficiencia cardíaca & 53 & 2,1 & $1,6-2,8$ \\
\hline EPOC & 82 & 3,4 & $2,6-4$ \\
\hline Uso previo de aspirina & 525 & 21 & $20-23$ \\
\hline \multicolumn{4}{|l|}{ Localización del infarto } \\
\hline Anterior & 905 & 37 & $35-39$ \\
\hline Inferior & 948 & 38 & $36-40$ \\
\hline Lateral & 110 & 4,4 & $4-5$ \\
\hline Indeterminado & 25 & 1 & $0,5-1,5$ \\
\hline \multicolumn{4}{|l|}{ Killip y Kimbal al ingreso } \\
\hline 1 & 1865 & 76 & $74-77$ \\
\hline$\|$ & 342 & 14 & $12-15$ \\
\hline III & 0 & & \\
\hline IV & 184 & 7,5 & $6,5-8,5$ \\
\hline
\end{tabular}

* $\mathrm{DE}=$ Desvío estándar

IC: Intervalo de confianza en las primeras 24 horas del IAM en el $89 \%$ de los casos; de estos, a su vez el $89 \%$ fueron angioplastias primarias, el $8 \%$ angioplastias de rescate y solo un $3 \%$ fueron a una estrategia farmacoinvasiva (Tabla 2).

El 96\% de las angioplastias primarias fueron exitosas y el $95 \%$ de los pacientes recibieron implantes de stents, de los cuales el $52 \%$ fueron liberadores de drogas. Las arterias responsables del infarto fueron las siguientes: descendente anterior (46\%), coronaria derecha $(36 \%)$, circunfleja $(13 \%)$, diagonal $(2 \%)$, tronco de coronaria izquierda (1\%) y puentes venosos $(0,6 \%)$.

En el $11 \%$ de los casos no se realizó tratamiento de reperfusión, fundamentalmente por la demora en la consulta.

\section{Demoras}

La demora desde el inicio de los síntomas hasta la admisión fue de 130 minutos (RIC 25-75: 60-305) en la población global. Cuando los pacientes fueron derivados de una institución a otra para completar su tratamiento, la demora hasta el segundo centro ue de 150 minutos (RIC 25-75: 60-360), mientras que la demora en los que consultaron directamente al centro donde fueron tratados, sin necesidad de ser transferidos, fue de 120 minutos (RIC 25-75: 60-266); $\mathrm{p}=0,05$. Más de $2 / 3$ de los sujetos ingresaron dentro de las 6 horas de comenzados los síntomas.

El tiempo puerta-balón de los pacientes a los que se les realizó angioplastia primaria fue de 131 minutos (RIC 25-75: 70-273), mientras que la ventana total de 
tiempo entre el inicio de los síntomas y el inflado del balón, considerado como el tiempo total de isquemia (TTI), fue de 315 minutos (RIC 25-75: 194-600) (Tabla 3 ). Solo el $35 \%$ tuvo un tiempo puerta-balón menor de 90 minutos.

Los pacientes que consultaron a un centro con posibilidad de realizar angioplastia tuvieron un tiempo puerta-balón de 100 minutos (RIC 25-75: 60-174), vs. 192 minutos (RIC 25-75: 98-395) en aquellos que debieron ser trasladados a otro centro para su tratamiento $(\mathrm{p}=0,0001)$. Esto tuvo impacto en el TTI: 259 minutos (RIC 25-75: 160-480) en los que no fueron transferidos y 414 minutos (RIC 25-75: 254-748) en los que debieron ser transferidos, $\mathrm{p}=<0,001$.

Siguiendo el mismo esquema y teniendo en cuenta que el número de pacientes fue menor, el tiempo puertaaguja de los que fueron tratados en el primer centro de consulta fue de 60 minutos (RIC 25-75: 35-95), vs. 65 minutos (RIC 25-75: 30-130) en los enfermos que tuvieron que ser derivados para realizar la infusión de fibrinolíticos $(p=0,2)$. Asimismo, no se encontraron diferencias estadísticamente significativas en la ventana total de tiempo, con 210 minutos (RIC 25-75: 110-360) vs. 180 minutos (RIC 25-75: 120-315), p = 0,35, para estos dos grupos, respectivamente. Solo el $20 \%$ de los pacientes fue tratado con fibrinolíticos dentro de los 30 minutos de su ingreso.

Se solicitó la opinión de los médicos tratantes respecto de las demoras al tratamiento y consideraron que hubo demoras en el tratamiento de reperfusión en 1208 pacientes (49\%), 60\% en los casos de angioplastia primaria y $62 \%$ en el uso de trombolíticos. También destacan el retraso de la consulta del paciente como uno de los factores más importantes. Si se toman en cuenta los tiempos ventana de los pacientes en los que hubo demoras y se los compara con el de aquellos en los que no se consideró que hayan existido demoras, hubo una diferencia de 3 horas en los casos de angioplastia primaria y de 90 minutos en los de reperfusión con trombolíticos.

El tratamiento médico indicado, tanto al ingreso como al alta, se encuentra en el material suplementario. El 97\% recibió aspirina al ingreso y el clopidogrel fue el inhibidor de los receptores P2Y12 más utilizado al alta en los tratados con angioplastia (69\%).

\section{Evolución intrahospitalaria}

En la Tabla 4 se detallan los eventos ocurridos durante la hospitalización. De los 65 pacientes $(2,6 \%)$ que tuvieron hemorragias, el $37 \%$ se vincularon a las punciones arteriales, pero todas ellas fueron hemorragias mínimas o menores. De las hemorragias mayores, 3 fueron cerebrales. La mediana de estadía hospitalaria fue de 4 días (RIC 25-75: 3-6); la de unidad coronaria también fue de 4 días (RIC 25-75: 2-5).

La mortalidad intrahospitalaria fue del 8,7\% $(\mathrm{n}=214$, la mayoría de causa cardíaca $(81 \%)$. Hemos observado que existe una fuerte correlación $(\mathrm{r}=0.71)$ entre mayor tiempo hasta la consulta y la mortalidad (Figura 1).

La mortalidad de los pacientes que ingresaron con insuficiencia cardíaca fue del $15 \%$, de los que ingresaron con shock cardiogénico, del $57 \%$ y de aquellos que ingresaron sin signos de insuficiencia cardíaca, del $2,6 \%$. La mortalidad de los tratados con trombolíticos fue del $9 \%$, vs. el 7,5\% registrado en los tratados con angioplastia, sin diferencias estadísticamente significativas $(\mathrm{p}=0,15)$. También se observó una relación entre la cantidad de horas desde el inicio de los síntomas hasta el ingreso institucional y la proporción de pacientes

\begin{tabular}{|lccc|}
\hline Reperfusión & $\mathbf{n}$ & $\mathbf{\%}$ & IC 95\% \\
\hline Reperfundidos & 2178 & 88 & $87-90$ \\
\hline Fibrinolíticos & 518 & 21 & $19-23$ \\
\hline Angioplastias realizadas en las primeras $24 \mathrm{~h}$ & 1938 & 89 & $87-90$ \\
\hline Angioplastia primaria & 1718 & 89 & \\
\hline Angioplastia de rescate & 149 & 8 & \\
\hline Angioplastia farmacoinvasiva & 67 & 3 & \\
\hline
\end{tabular}

Tabla 2. Reperfusión

IC: Intervalo de confianza

\begin{tabular}{|lcc|}
\hline Tiempos & Mediana & RIC 25-75\% \\
\hline Dolor-consulta & 101 & $50-240$ \\
\hline Dolor-ingreso institucional & 130 & $60-305$ \\
\hline Primer contacto médico-realización primer ECG & 10 & $1-10$ \\
\hline Puerta-aguja & 60 & $33-120$ \\
\hline Ventana puerta-aguja & 190 & $115-330$ \\
\hline Puerta-balón & 131 & $70-273$ \\
\hline Ventana puerta-balón & 315 & $194-600$ \\
\hline
\end{tabular}

RIC: Rango intercuartilo 
Tabla 4. Eventos intrahospitalarios

\begin{tabular}{|c|c|c|c|}
\hline Eventos & $\mathbf{n}$ & $\%$ & IC $95 \%$ \\
\hline Mortalidad general & 214 & 8,7 & $8-10$ \\
\hline Mortalidad cardiovascular & 174 & 7 & $6-8$ \\
\hline Angina posinfarto & 60 & 2,4 & $1,9-3$ \\
\hline Reinfarto & 33 & 1,3 & $0,9-1,9$ \\
\hline Accidente cerebrovascular & 24 & 1 & $0,6-1,4$ \\
\hline Fibrilación auricular & 98 & 4 & $3-5$ \\
\hline Paro cardíaco & 235 & 9,5 & $8,4-10$ \\
\hline \multicolumn{4}{|l|}{ Ritmo en el paro cardíaco } \\
\hline Fibrilación / Taquicardia ventricular & 132 & 56 & \\
\hline Actividad eléctrica sin pulso & 33 & 14 & \\
\hline Asistolia & 53 & 23 & \\
\hline No documentado & 17 & 7 & \\
\hline Hemorragia & 65 & 2,6 & $2-3,3$ \\
\hline Mínima & 34 & 52 & \\
\hline Moderada & 14 & 22 & \\
\hline Mayor & 17 & 26 & \\
\hline Insuficiencia cardíaca en la evolución & 289 & 12 & $10-13$ \\
\hline Shock cardiogénico & 220 & 9 & $8-10$ \\
\hline Complicaciones mecánicas & 28 & 1,1 & $0,7-1,6$ \\
\hline Comunicación interventricular & 17 & 60 & \\
\hline Insuficiencia mitral & 9 & 32 & \\
\hline Ruptura cardíaca externa & 2 & 7 & \\
\hline
\end{tabular}

IC: Intervalo de confianza
Fig. 1. Correlación entre horas hasta el momento del ingreso y muerte (coeficiente de correlación $=0,71$ )

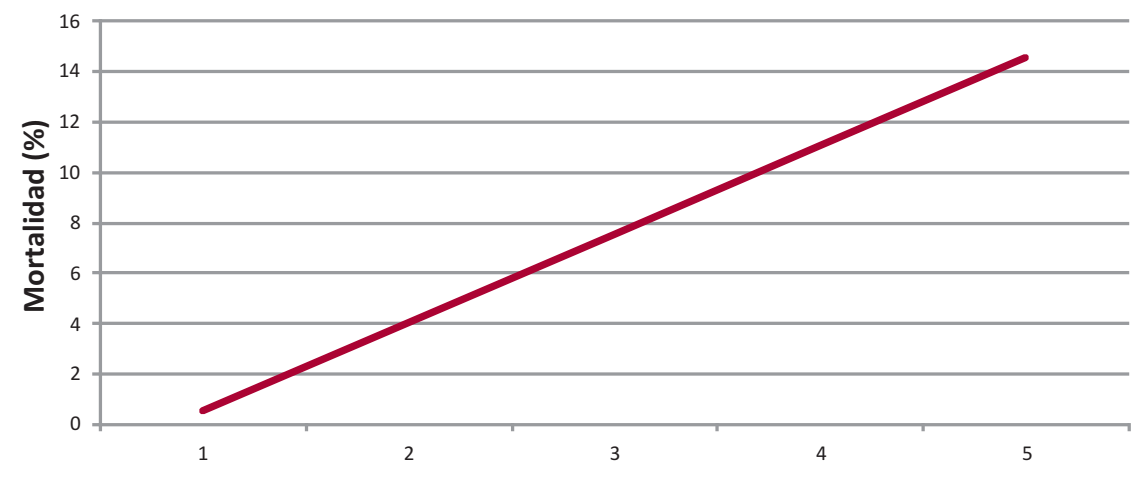

Tiempo dolor consulta: $1=1-3 \mathrm{~h} ; 2$ = 3-6 h; $3=6-12 \mathrm{~h} ; 4=12-24 \mathrm{~h} ; 5>24 \mathrm{~h}$ con deterioro grave de la fracción de eyección (FEy) del ventrículo izquierdo, medida en forma cualitativa durante la internación: 0-3 horas, 9,5\%; 3-6 horas, 11,5\%; 6-12 horas, 16\%, 12-24 horas, $19 \%$; y más de 24 horas, $21 \%$ ( $\mathrm{p}=0,02)$.

En el análisis multivariado de regresión logística, las variables independientes asociadas a mortalidad de modo estadísticamente significativo fueron la edad, el sexo femenino, la presencia de shock cardiogénico al ingreso y la falta de reperfusión. Que el paciente sea tratado en un centro con hemodinamia no se asoció con una disminución de la mortalidad (ver material suplementario).

\section{DISCUSIÓN}

El registro continuo ARGEN-IAM-ST incluyó casi 2500 pacientes, y, aunque se redujo el número de centros participantes y provincias incluidas, los niveles de complejidad de las instituciones participantes son similares a los de la encuesta nacional del año 2015. (3) 
El análisis nos muestra tasas de reperfusión de casi el $90 \%$, una elevada utilización de angioplastia primaria, y, a pesar de que la mitad de los pacientes debieron ser transferidos a centros de mayor complejidad, solo un 3\% recibió una estrategia de reperfusión farmacoinvasiva y el $8 \%$ angioplastia de rescate. La mortalidad global fue del 8,7\%, niveles muy similares a los de encuestas previas y de registros publicados por centros con residencias en cardiología. (4,5) Juan P. Costabel, Nicolás González, Ezequiel Zaidel, Marcela Altamirano, Miguel Schiavone, cols; Investigadores del Consejo Argentino de Residentes de Cardiología (CONAREC La mortalidad es más elevada que la de algunos registros internacionales, como el francés, pero similar a la de los registros públicos de ese país y menor que la de los registros estadounidenses. (6)

Aunque un elevado porcentaje de los pacientes consulta con menos de 6 horas de evolución de los síntomas, los que reciben el tratamiento con tiempos puerta-balón y puerta-aguja según las recomendaciones de guías de práctica clínica son inapropiados. Este punto requiere gran cooperación y organización para revertir la situación, ya que, como vimos, en el registro se aprecia la relación lógica de que a mayor tiempo hasta la reperfusión, mayor mortalidad, y una proporción más elevada de pacientes conviven con un deterioro grave de la FEy, independientemente del método de reperfusión que se haya utilizado, por lo que, si se administrara en tiempo y forma, la reperfusión evitaría dichas complicaciones.

Los estudios que analizaron la estrategia de administrar trombolíticos en el sitio de derivación versus el traslado para angioplastia primaria no mostraron beneficio cuando el tiempo de evolución de los síntomas era menor de 3 horas, pero el traslado resultó beneficioso con tiempos mayores, sobre todo, en términos de incidencia de reinfarto y accidente cerebrovascular. (7) Sin embargo, los tiempos puerta-balón de esos estudios fueron muy bajos y muy difíciles de trasladar a la vida real, por lo que, teniendo en cuenta estas cifras, un elevado número de pacientes podría beneficiarse con la utilización de fibrinolíticos antes de la derivación. (8)

En más del $60 \%$ de los infartos, los médicos detectan demoras en el tratamiento, sobre todo atribuidas a retraso en la consulta del paciente y la necesidad de derivar a otro centro para angioplastia. Este aspecto pudo comprobarse, dado que la medición el TTI para la angioplastia fue mayor en este grupo. Es evidente, entonces, la necesidad de implementar mejores estrategias dirigidas a la población para promover la consulta temprana. Otro punto clave es la educación y el suministro de medios adecuados para el diagnóstico y el tratamiento precoz en el lugar de la consulta, o a través de un sistema de redes, antes de la eventual derivación a centros de mayor complejidad.

El análisis de las variables predictoras independientes de mortalidad coincide con lo que muestra la literatura y con subestudios realizados previamente por autores del ARGEN-IAM-ST. (9-11) En 2020, se cumplen cinco años del registro ARGEN-IAM-ST, el más extenso hasta el momento en la Argentina. Este registro cumple con uno de sus principales objetivos: brindar información permanente sobre el IAM, para discutir su atención y mejorar la calidad de los lineamientos que proponen las sociedades científicas a través de sus guías y consensos de práctica clínica, dada la considerable morbimortalidad de esta afección. (12)

\section{Limitaciones}

El registro continuo presenta algunas diferencias estructurales con la encuesta inicial, ya que, si bien presenta un mayor número de casos, que lo posiciona como el registro argentino de IAM de mayor alcance en la actualidad, esto se obtuvo en un tiempo más prolongado y con poco menos del $30 \%$ de los centros que participaron en el primer registro de ARGEN-IAM-ST. Esto hace que la mayoría de los casos se concentren en las instituciones más grandes, tanto públicas como privadas, y podría haber un sesgo en los centros de menor complejidad. Por otro lado, la falta de auditoría de los datos en los centros, por falta de apoyo económico, podría generar interrogantes sobre el registro del dato. A pesar de ello, las instituciones que participan mantienen un alto grado de compromiso con el registro, y, más aún, con esta importante patología cardiológica.

\section{CONCLUSIONES}

Los datos actuales del registro continuo de IAM en la Argentina muestran resultados similares a los de la encuesta de 2015, con una alta tasa de reperfusión (88\%) y una mortalidad intrahospitalaria del 8,7\%. De la misma manera, las demoras al tratamiento aún son importantes, especialmente en el tiempo de consulta de los pacientes y en la derivación a otros centros, con gran influencia en los tiempos totales de isquemia.

En este segundo reporte de resultados podemos observar de manera concordante la situación del IAM en la Argentina, lo que nos permite trabajar en las metas propuestas por las sociedades científicas para lograr una mejora en la calidad de atención.

\section{Conflicto de intereses}

El trabajo presentado es independiente. Los autores declaran que no poseen conflicto de intereses.

(Véase formulario de conflicto de intereses de los autores en la web / Material suplementario).

\section{BIBLIOGRAFÍA}

1. Ibanez B, James S, Agewall S, et al. 2017 ESC Guidelines for the management of acute myocardial infarction in patients presenting with ST-segment elevation: The Task Force for the management of acute myocardial infarction in patients presenting with ST-segment elevation of the European Society of Cardiology (ESC). Eur Heart J 2018;39:119-77. https://doi.org/10.1093/eurheartj/ehx393

2. Bhatnagar P, Wickramasinghe K, Williams J, Rayner M, Townsend N. The epidemiology of cardiovascular disease in the UK 2014. Heart 2015;101:1182-9. https://doi.org/10.1136/heartjnl-2015-307516 
3. Gagliardi JA, Charask A, Perna E, D’Imperio H, Bono J, Castillo Costa Y y cols. National Survey of ST-Segment Elevation Acute Myocardial Infarction in Argentina (ARGEN-IAM-ST). Rev Argent Cardiol 2016;84:524-33. https://doi.org/10.7775/rac.es.v84.i6.9508

4. Higa CC, D’Imperio H, Blanco P, Charask A, Cohen Arazi H, Novo F, cols; On Behalf of the investigators of the SCAR and ARGEN-IAMST registries. Comparación de dos registros argentinos de infarto de miocardio: SCAR 2011 y ARGEN-IAM ST 2015. Rev Argent Cardiol 2019;87:19-25.

5. Pérez GE, Costabel JC, González N, Zaidel E, Altamirano M, Schiavone M, cols; Investigadores del Consejo Argentino de Residentes de Cardiología (CONAREC). Infarto agudo de miocardio en la República Argentina. Registro CONAREC XVII. Rev Argent Cardiol 2013;81:390-9. https://doi.org/10.7775/rac.es.v81.i5.1391

6. Massoullie G, Wintzer-Wehekind J, Chenaf C, Mulliez A, Pereira B, Authier N, et al. Prognosis and management of myocardial infarction: Comparisons between the French FAST-MI 2010 registry and the French public health database. Arch Cardiovasc Dis 2016;109:303-10. https://doi.org/10.1016/j.acvd.2016.01.012

7. Widimský P. Long distance transport for primary angioplasty vs immediate thrombolysis in acute myocardial infarction Final results of the randomized national multicentre trial-PRAGUE-2. Eur Heart J 2003;24:94-104. https://doi.org/10.1016/S0195-668X(02)00468-2

8. Andersen HR, Nielsen TT, Vesterlund T, Grande P, Abildgaard U,
Thayssen P, et al. Danish multicenter randomized study on fibrinolytic therapy versus acute coronary angioplasty in acute myocardial infarction: rationale and design of the danish trial in acute myocardial infarction-2 (DANAMI-2). Am Heart J 2003;146:234-41. https://doi. org/10.1016/S0002-8703(03)00316-8

9. Castillo Costa YB, Caccavo A, Charask A, Moreno K, Cassano C, Gagliardi JA. Características de los pacientes mayores de 75 años en el Registro ARGEN-IAM-ST. Rev Argent Cardiol 2019,87;48-52. https://doi.org/10.7775/rac.es.v87.i1.12558

10. Macín SM, Sueldo M, Perna ER, Tajer CD, Cerezo GH, Struminger M y cols. Características clínicas y evolución hospitalaria de mujeres con Infarto agudo de miocardio en el Registro Nacional de Infarto SAC-FAC. Rev Fed Arg Cardiol 2018;47:125-9.

11. Charask AA, Castillo Costa YB, D'Imperio H, Perna ER, Zapata G, Tajer CD y cols. Pacientes con infarto agudo de miocardio con elevación del ST trasladados a centros con hemodinamia. Encuesta Nacional de Infarto Agudo de Miocardio con Elevación del ST en la República Argentina (ARGEN-IAM-ST). Rev Argent Cardiol 2017;85:90-102.

12. Blanco P, Borracci RA, Giorgi M, Higa C, Botto F, Juan Gagliardi $\mathrm{J}$, por los Investigadores del Área de Investigación SAC y el Consejo de Emergencias SAC. Años de vida perdidos por infarto agudo de miocardio en la Argentina entre 1991 y 2005. Rev Argent Cardiol 2008;76:442-9. 


\section{MATERIAL SUPLEMENTARIO}

\section{APÉNDICE}

\section{Lista de centros participantes y responsables de centro}

Hospital Gral. de Agudos Dr. Cosme Argerich - Luciana Puente

Instituto Cardiovascular de Rosario - Gerardo Zapata

Hospital San Juan de Dios de La Plata - Oscar Pisana/Diego Echazarreta

Sanatorio Güemes - Ricardo Villareal

Clínica Santa Isabel - Víctor Mauro/Yanina Castillo Costa

Clínica Bazterrica - Carlos Barrero/ Adrián Charask

Sanatorio San Carlos - Matías Calandrelli

Sanatorio Allende Nueva Córdoba - Julio Bono

Centro Privado de Cardiología - Eduardo G. Hasbani

Instituto de Cardiología J. F. Cabral - Stella Macín/Facundo Falcón

Centro Modelo de Cardiología - Juan Muntaner

Hospital El Cruce "Dr. Néstor Kirchner" - Tomás Vassia

Hospital Luis Lagomaggiore - Jorge Piasentin

Sanatorio Privado Gatti - Pablo Moreno

Sanatorio Pasteur - María Pía Marturano

Sanatorio Juan XXIII - Roberto Bernardini/Nicolás Menichini

Hospital para la Comunidad de Arias - Joaquín Sangiorno

Centro de Alta Complejidad - Pablo Agüero

Hospital Dr. Raúl F. Larcade - Gabriel Jans

Hospital Gral. de Agudos "Juan A. Fernández" - Patricia Guitelman

Hospital San José de Pergamino - Luis Bahamonde

Hospital Gral. de Agudos "Dr. T. Álvarez" - Daniel H. Avayu/Marcos P. Tomasella

Hospital Universitario Austral - Horacio Fernández

Clínica de Cuyo - Ariel Baigorria Jayat/María Elisa de la Fuente

Hospital Subzonal "Dr. Andrés R. Isola" - Norman Casado

Hospital Dr. Guillermo Rawson - Adrián H. D’Ovidio

Sanatorio de la Ciudad, Puerto Madryn - Julián Tiranti

Hospital Artémides Zatti - José Luis Rovasio/Silvia Framarini

Instituto de Cardiología Dr. González Sabathié - Antonio Gentile/Mario Ciafardoni

Hospital Español de Buenos Aires - Liliana Nicolisi

Sanatorio Fueguino de Diagnóstico y Tratamiento - Mauro Dotto/Raúl E. Figueroa

Hospital de San Bernardo - Augusto Barbosa

Fundación Médica de Rio Negro y Neuquén - Demetrio Thalasselis

Instituto. Modelo de Cardiología Privado de Córdoba. - Eduardo Conci/Walter Quiroga

Hospital Italiano de Córdoba - Fernando Gragera

Hospital Ramón Carrillo - David Marcelo Krivich

Hospital Córdoba - Marcos De la Vega

Clínica y Maternidad Suizo Argentina - Juan Caros Medrano

Hospital San Felipe San Nicolás - Raúl Alejandro Quijano

Hospital El Carmen, Mendoza - Oscar Fernando Vidal

Clínica Universitaria Reina Fabiola, Córdoba - Raúl Jesús Barcudi

Clínica Pasteur SA, Neuquén - Claudio Ploger/Ana Duret

Hospital Gral. de Agudos Dr. Zubizarreta - José María Soler

Sanatorio San Martín, Venado Tuerto, Santa Fe - Javier Matcovik

Sanatorio de la Trinidad, San Isidro, Bs. As. - Juan Taccari/Walter Nieto

Hospital Italiano de Buenos Aires, CABA - José Luis Navarro Estrada/Francisco José Romeo

Hospital Británico de Buenos Aires, CABA - Horacio Alberto Avaca/Mauro Gastón Gingins

Hospital Mi Pueblo, Florencio Varela, Buenos Aires - Santiago Tur/Federico Bodega

Hospital Pablo Soria - Franz Rivero Paz

Sanatorio Allende Cerro, Córdoba - Roberto Miguel A. Colque

Hospital Privado del Sur - Raúl Cermesoni/Marcelo Guimaraenz

Hospital Privado de la Comunidad de Mar del Plata - Álvaro Facta

Hospital General de Agudos Dr. Ramos Mejía - Justo Cabrales 
Hospital Luisa C. de Gandulfo - Juan Pullido

Clínica San Martín - Pablo Maldonado

Hospital Italiano de La Plata - Cecilia Beltrano

Hospital Iriarte - David Parisi

HIGA Gral. San Martín - Luis Medesani

HIGA Rossi - Carlos Martínez

Hospital Pirovano - Ricardo Mejail

Hospital Español de Rosario - Daniel Edgardo Miraglia

Clínica Yunes - Edgar Aguilar

Sanatorio Modelo Quilmes - Adrián Hrabar/Alberto Fernández

Sanatorio Ntra. Sra. del Rosario - Gustavo Bustamante Labarta

Hospital Teodoro J. Schestakow - Leonardo Schiavone

Hospital Dr. J. M. Valdano - Ramiro Alberto Astegiano

IOT - Oscar Ariel Vogel

Hospital Héctor Cura, Olavarría - Ernesto Ylarri

Policlínico Regional Juan D. Perón - Sandra Mugnaini

Policlínico Modelo de Cipolletti -Diego Figoni

RAPIAM (Red de Atención Prov. del IAM La Rioja) - Horacio Pomés Iparaguirre

Sanatorio Los Lapachos de Jujuy - Luis Freijo

Hospital Lamadrid de Monteros - Andrea Piredda

Clínica Del Valle - Miguel Salva

Hospital Zonal Bariloche - Germán Santamaría

Hospital de Alta Complejidad J. D. Perón - Christian Smith/Nicolás Areco

Hospital L. Molas, Santa Rosa, La Pampa - Fabián Kubaruk

Sanatorio Mitre - Hernán Cohen Arazi 


\begin{tabular}{|lcccc|}
$\begin{array}{l}\text { Horas hasta } \\
\text { el ingreso }\end{array}$ & $\mathbf{N}$ & $\%$ & Mediana (minutos) & RIC 25-75 \\
\hline $0-3$ & 1449 & 61 & 75 & $40-120$ \\
\hline$>3-6$ & 438 & 18 & 250 & $219-300$ \\
\hline$>6-12$ & 295 & 12 & 500 & $420-600$ \\
\hline$>12-24$ & 144 & 6 & 1020 & $855-1225$ \\
\hline$>24$ & 75 & 3 & 1897 & $1599-2520$ \\
\hline
\end{tabular}

Tabla 5. Distribución de los pacientes según intervalos de tiempo (preestablecidos) desde el comienzo de los síntomas hasta el ingreso a la institución.

\begin{tabular}{|lccc|}
\hline & $\mathbf{n}$ & $\mathbf{\%}$ & IC 95\% \\
\hline Hubo demoras en el tratamiento & 1208 & 49 & $47-51$ \\
\hline Consulta del paciente & 917 & 75 & $73-78$ \\
\hline Retraso de ambulancia & 524 & 43 & $41-46$ \\
\hline Guardia & 359 & 30 & $27-32$ \\
\hline Falla médica en diagnóstico & 312 & 26 & $23-28$ \\
\hline Demora de hemodinamia & 281 & 23 & $21-26$ \\
\hline Problemas administrativos & 86 & 7 & $6-9$ \\
\hline ECG dudoso inicial & 108 & 9 & $7-11$ \\
\hline Paro cardíaco & 64 & 5 & $4-7$ \\
\hline Otros motivos & 87 & 7 & $6-9$ \\
\hline
\end{tabular}

Tabla 6. Detalle de las causas de las demoras

Tabla 7. Tratamientos instaurados

\begin{tabular}{|c|c|c|c|c|c|c|}
\hline Fármacos & \multicolumn{3}{|c|}{$\begin{array}{c}\text { Al ingreso } \\
n=2464\end{array}$} & \multicolumn{3}{|c|}{$\begin{array}{c}\text { Al alta } \\
n=2250\end{array}$} \\
\hline Aspirina & 2387 & 97 & $96-98$ & 1966 & 87 & $86-89$ \\
\hline Clopidogrel & 1803 & 73 & $71-75$ & 1415 & 63 & $61-65$ \\
\hline Prasugrel & 162 & 6,5 & $5-8$ & 152 & 7 & $6-8$ \\
\hline Ticagrelor & 410 & 17 & $15-18$ & 363 & 16 & $15-18$ \\
\hline Inhibidores GPIIbIIla & 134 & 5 & $4-6$ & & & \\
\hline \#HNF anticoagulante & 821 & 33 & $31-35$ & & & \\
\hline$\infty \mathrm{HBPM}$ anticoagulante & 647 & 26 & $24-28$ & & & \\
\hline Bivalirudina & 26 & 1 & $0,6-1,5$ & & & \\
\hline Beta-bloqueantes & 1235 & 50 & $48-52$ & 1684 & 75 & $73-77$ \\
\hline$\Omega \mathrm{IECA}$ & 1110 & 45 & $43-47$ & 1279 & 57 & $56-59$ \\
\hline ФARA ॥ & 154 & 6 & $5-7$ & 208 & 9 & 8-10 \\
\hline Estatinas & 2191 & 89 & $88-90$ & 1882 & 84 & $82-85$ \\
\hline Antialdosterónicos & 273 & 11 & $10-12$ & 380 & 17 & $15-18$ \\
\hline Acenocumarol-warfarina & 47 & 2 & $1,4-2,5$ & 89 & 4 & $3-5$ \\
\hline
\end{tabular}

\# HNF anticoagulante: heparina no fraccionada en dosis anticoagulante; $\infty$ HBPM anticoagulante: heparina de bajo peso molecular en dosis anticoagulante; SIECA: inhibidores de la enzima convertidora de angiotensina; ФARA II: antagonistas de los receptores de angiotensina II 
Tabla 8. Distribución de casos por provincias

\begin{tabular}{|lccc|}
\hline Provincias & $\mathbf{N}^{\circ}$ de centros & $\begin{array}{c}\mathbf{N}^{\circ} \text { de } \\
\text { IAM incluidos }\end{array}$ & $\%$ \\
\hline Buenos Aires & 22 & 527 & 25,0 \\
\hline CABA & 13 & 613 & 21,9 \\
\hline Catamarca & 1 & 161 & 6,5 \\
\hline Chubut & 3 & 40 & 1,6 \\
\hline Córdoba & 9 & 127 & 5,1 \\
\hline Corrientes & 1 & 301 & 12,2 \\
\hline Formosa & 1 & 17 & 0,7 \\
\hline Jujuy & 3 & 36 & 1,5 \\
\hline La Pampa & 1 & 1 & 0,0 \\
\hline La Rioja & 1 & 105 & 4,3 \\
\hline Mendoza & 4 & 47 & 1,9 \\
\hline Misiones & 1 & 6 & 0,2 \\
\hline Neuquén & 1 & 47 & 1,9 \\
\hline Río Negro & 6 & 79 & 3,2 \\
\hline Salta & 1 & 14 & 0,57 \\
\hline San Juan & 1 & 42 & 1,7 \\
\hline Santa Fe & 1 & 252 & 10,2 \\
\hline Santiago del Estero & 5 & 2 & 0,1 \\
\hline Tierra del Fuego & 1 & 0,1 \\
\hline Tucumán & 1 & 2464 \\
\hline Total & 1 & 100 \\
\hline
\end{tabular}

Tabla 9. Regresión logística, predictores de mortalidad

\begin{tabular}{lccc}
\hline *Variables & OR & P & IC 95\% \\
\hline Edad & 1,035 & 0,000 & $1,02-1,05$ \\
\hline Sexo & 0,62 & 0,004 & $0,45-0,86$ \\
\hline Shock al ingreso & 21 & 0,000 & $15-31$ \\
\hline Reperfusión & 0,48 & 0,000 & $0,35-0,73$ \\
\hline Centro con hemodinamia & 0,734 & 0,148 & $0,48-1,11$
\end{tabular}

Codificación de las variables: edad, variable continua; sexo: $0=$ femenino, $1=$ masculino; shock al ingreso: $0=\sin$ shock, 1 = con shock; reperfusión: $0=$ no recibió, $1=$ recibió; centro con hemodinamia: $0=$ no, $1=$ sí 